\title{
Détermination de la masse volumique du solide pour un échantillon de sol argileux : validité de la méthode utilisant les propriétés de retrait de l'échantillon remanié sous dessication pneumatique
}

\author{
A. Bruand ${ }^{1}$, V. Sorani ${ }^{2}$ et M. Hardy ${ }^{1}$ \\ avec la collaboration technique de $H$. Gaillard ${ }^{1}$
}

1 Service d'Etude des Sols et de la Carte pédologique de France, INRA, Ardon, 45160 Olivet

${ }^{2}$ Chaire de Pédologie, INA PG, 78850 Thiverval-Grignon, France

(reçu le 1-3-1988, accepté le 28-9-1988)

Résumé - La masse volumique du solide $\left(\rho_{\mathrm{s}}\right)$ déterminée à partir des propriétés de retrait est inférieure à celle obtenue en pycnométrie à gaz. Cette différence est expliquée par le piégeage d'air dans les échantillons après les avoir soumis à une pression pneumatique.

densité - matériau argileux - pycnométrie à gaz

Summary - Particle density in a clayey soil sample : validity of the method utilising the shrinkage properties of the sample remoulded by air pressure equipment. The particle density value $\left(\rho_{\mathrm{s}}\right)$ calculated from the shrinkage curve is lower than that obtained by using a gas pycnometer. This difference is explained by the presence of air trapped inside the sample after subjecting it to pneumatic pressure.

particle density - clayey material - gas pycnometry

\section{Introduction}

La connaissance de la masse volumique de la phase solide de l'échantillon $\left(\rho_{s}\right)$ est nécessaire pour de nombreux travaux en science du sol, notamment pour rapporter les résultats au volume de la phase solide (Tessier, 1984; Robain et Curmi, 1986) ou effectuer des bilans volumiques lors de l'analyse des systèmes de porosité (Fiès, 1984; Chrétien, 1986; Sorani et al., 1987). Pour les matériaux argileux, une méthode permettant de déterminer $\rho_{s}$ consiste à étudier les variations du volume massique de l'échantillon $\left(V_{m}\right)$, ou volume apparent, en fonction de son volume d'eau massique $\left(V_{e}\right)$. Dans ce but, l'échantillon subit un remaniement qui permet de l'étudier dans un domaine de valeur de $V_{e}$ où, d'une part, la totalité de l'espace poral est occupée par de l'eau (état saturé) (Bruand, 1986) et, d'autre part, la détermination de $\mathrm{V}_{\mathrm{m}}$ à l'aide de la méthode au pétrole est possible (Monnier et al., 1973). La courbe de retrait (Haines, 1923; Reeve et Hall, 1978), qui représente $V_{m}$ en fonction de $V_{e}$, est alors une droite de pente égale à 1 (droite de saturation) puisque :

$$
\mathrm{V}_{\mathrm{m}}=\mathrm{V}_{\mathrm{s}}+\mathrm{V}_{\mathrm{e}}
$$

avec $V_{s}$ constant et représentant le volume massique du solide égal à $1 / \rho_{\mathrm{s}}$.

L'objectif de cette note est de discuter la validité d'une telle méthode de détermination de $\rho_{\mathrm{s}}$ après avoir comparé cette valeur à celle obtenue en pycnométrie à gaz. 


\section{Matériel}

Le matériau de sol étudié provient de l'horizon $B_{t}$ argileux d'un profil de "terres d'Aubues". II s'agit de sols de type lessivé acide (Rhodic luvisol) développés sur les plateaux calcaires du sud-est du Bassin parisien (Baize, 1972) (Tableau 1). Une partie de ce matériau est conservée humide à une température voisine de $4^{\circ} \mathrm{C}$, l'autre est séchée à l'air.

\section{Méthodes}

Détermination de $\rho$ s à partir de la droite de saturation

Le matériau conservé humide est transformé en une pâte homogène en le dispersant dans l'eau par agitation mécanique. Le mélange obtenu ( 3 volumes d'eau pour 1 d'échantillon) est centrifugé, puis malaxé de façon à obtenir une pâte contenant $0,5 \mathrm{~cm}^{3} / \mathrm{g}$ d'eau. Celle-ci est versée dans des anneaux en PVC ( $32 \mathrm{~mm}$ de diamètre sur $10 \mathrm{~mm}$ de hauteur), puis soumise à une pression pneumatique à l'aide des dispositifs décrits par Richards (1941) et Tessier (1984). Les différentes valeurs de $V_{e}$ sont obtenues en faisant varier la pression appliquée entre $10^{3}$ et $10^{4} \mathrm{hPa}$. $\mathrm{V}_{\mathrm{m}}$ est déterminé par la méthode au pétrole (Monnier et al., 1973). $V_{m}$ et $V_{e}$ sont rapportés à la masse après séchage à l'étuve à $105^{\circ} \mathrm{C}$ pendant 12 h et exprimés en $\mathrm{cm}^{3} / \mathrm{g}$. Le volume massique du solide $\left(V_{s}\right)$ correspond à la valeur moyenne calculée à partir de $n$ couples de valeurs de $V_{m}$ et $V_{e}$, à l'aide de la relation :

$$
V_{s}=\underline{\Sigma\left(V_{m}-V_{e}\right)}
$$

$$
\text { n }
$$

$V_{S}$ est l'ordonnée à l'origine de la droite de retrait moyenne de pente égale à 1 (Fig. 1).

\section{Détermination de $\rho_{s}$ en pycnométrie à gaz}

Les déterminations sont effectuées, à la température de $20^{\circ} \mathrm{C}$, en utilisant de l'hélium dans un pycnomètre 1305

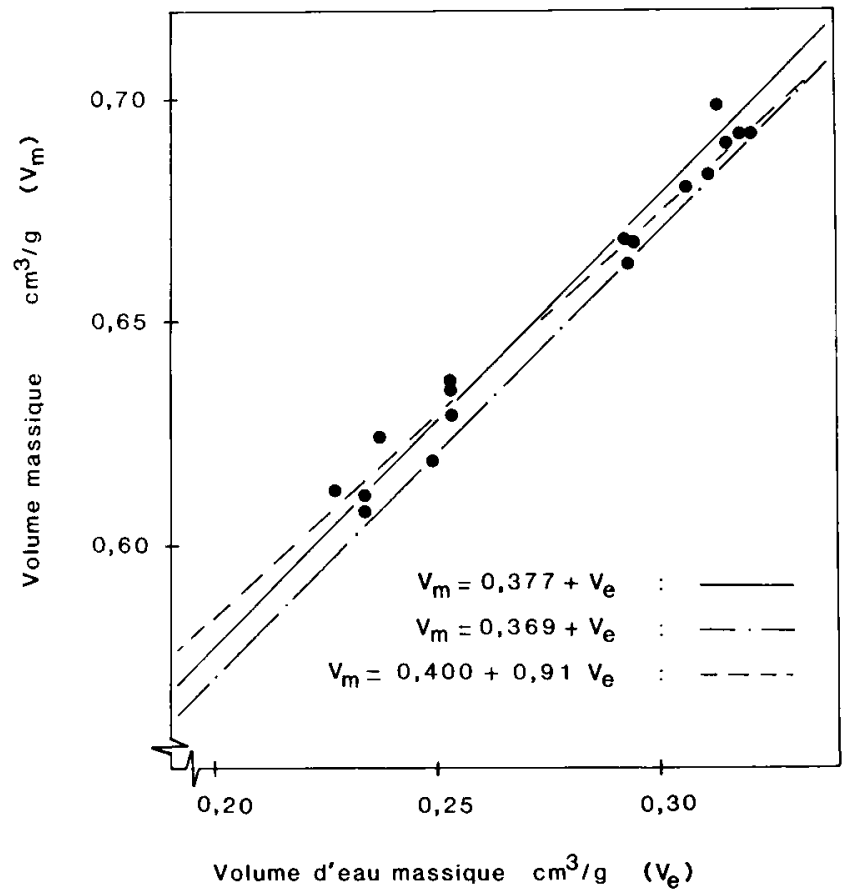

Fig. 1. Relations entre $v_{m}$ et $v_{e}$ : droite de retrait normal moyenne (-), droite de retrait normal minorante au nuage de points (-. - ) et droite de régression de $V_{m}$ en $v_{e}(--)$.

de chez Micromeretics. Le matériau séché à l'air est forcé à travers un tamis de $2 \mathrm{~mm}$, séché à l'étuve à $105^{\circ} \mathrm{C}$ pendant $12 \mathrm{~h}$ puis ramené à la température ambiante. Trois répétitions sont faites avec des prises d'essai de $3 \mathrm{~g}$.

\section{Résultats et Discussion}

Les valeurs de $V_{m}$ en fonction de $V_{e}$ sont présentées dans la Figure 1. $\mathrm{V}_{\mathrm{s}}$, calculé à l'aide de la relation $(2)(n=17)$, est égal à $0,377 \pm 0,003$ $\mathrm{cm}^{3} / \mathrm{g} \cdot \rho_{\mathrm{s}}$ est alors égal à $2,65 \pm 0,02 \mathrm{~g} / \mathrm{cm}^{3}$. Cette valeur est inférieure à celle obtenue en pycnométrie à gaz, puisque dans ce cas $\rho_{\mathrm{s}}=2,72 \pm 0,01$ $\mathrm{g} / \mathrm{cm}^{3}$.

\begin{tabular}{|c|c|c|c|c|c|c|c|}
\hline & \multicolumn{4}{|c|}{ Granulométrie en \% pondéral } & \multirow{2}{*}{$\begin{array}{l}\text { Carbone } \\
\text { organique } \\
\mathrm{g} / 100 \mathrm{~g}\end{array}$} & \multirow{2}{*}{$\begin{array}{l}\text { CEC } \\
\text { meq } / 100 \mathrm{~g}\end{array}$} & \multirow{2}{*}{$\begin{array}{c}\begin{array}{c}\text { Surface } \\
\text { externe }\end{array} \\
N_{2} \\
m^{2} / g\end{array}$} \\
\hline$<2 \mu m$ & $\begin{array}{l}2 \\
20 \mu m\end{array}$ & $\begin{array}{l}20 \\
50 \mu m\end{array}$ & $\begin{array}{l}50 \\
200 \mu \mathrm{m}\end{array}$ & $\begin{array}{l}200 \\
2000 \mu \mathrm{m}\end{array}$ & & & \\
\hline 55,7 & 26,1 & 16,2 & 1,7 & 0,3 & 0,3 & 22 & 61 \\
\hline
\end{tabular}

Tableau I. Résultats des analyses physique et chimique. 
Cette différence peut être expliquée par la présence d'air piégé dans les échantillons remaniés sous forme de pâte (Cabidoche et al., 1985) et ceci bien que la valeur de pression correspondant au point d'entrée d'air n'ait pas été atteinte (Bruand et Prost, 1987). Cette hypothèse énoncée, il reste à expliquer pourquoi de l'air peut se trouver piégé dans la pâte. Une première possibilité est l'incorporation de bulles d'air au cours de la préparation de la pâte lors de l'agitation mécanique. La liquidité du mélange obtenu avec les proportions employées n'est pas en faveur de cette explication. Une autre explication fait intervenir la dissolution des gaz dans l'eau décrite par la loi de Henry (Hillel, 1988). A la pression atmosphérique et à $20^{\circ} \mathrm{C}$, un litre d'eau contient environ $19 \mathrm{~cm}^{3}$ d'air à l'état dissous et l'on peut admettre que la quantité dissoute croit proportionnellement avec la pression entre la pression atmosphérique et $10^{4} \mathrm{hPa}$ (Violle, 1884).

Dans le dispositif utilisé pour fixer le potentiel matriciel de l'eau, une fois l'équilibre atteint, l'échantillon est ramené à la pression atmosphérique. L'eau présente dans cet échantillon libère alors un volume de gaz qui est, à température constante, fonction de la quantité d'eau et de la pression appliquée. A $10^{4} \mathrm{hPa}$, la quantité de gaz qui est libérée lorsque l'on ramène l'échantillon à la pression atmosphérique peut être estimée à $180 \mathrm{~cm}^{3}$ par litre d'eau. Si une partie de ce volume ne peut être évacuée de l'échantillon, elle reste piégée jusqu'à la mesure de $V_{m}$ à l'aide de la méthode au pétrole. Pour chaque échantillon, la masse à $105^{\circ} \mathrm{C}$ et la teneur en eau à $10^{4} \mathrm{hPa}$ sont respectivement voisines de $4 \mathrm{~g}$ et de $0,25 \mathrm{~cm}^{3} / \mathrm{g}$. On peut alors retenir $1 \mathrm{~cm}^{3}$ comme volume d'eau présent à $10^{4} \mathrm{hPa}$ dans l'échantillon. II se forme $0,18 \mathrm{~cm}^{3}$ de gaz dans celui-ci lorsqu'il est ramené à la pression atmosphérique. II suffit alors que seulement $1 / 5$ de ce volume demeure piégé pour expliquer la différence de $\rho_{\mathrm{s}}$ enregistrée précédemment.

La pente de la droite de régression de $V_{m}$ en $V_{e}$, qui est de $0,91\left(r^{2}=0,99 ; n=17\right)$ (Fig. 1), traduit cette incorporation croissante d'air dans l'échantillon lorsqu'il est ramené à la pression atmosphérique. Une détermination de $V_{s}$ permettant de minimiser ce biais expérimental consiste à prendre la droite de pente égale à 1 minorante au nuage de point présenté Figure 1. Le calcul donne $\rho_{\mathrm{s}}=2,71 \mathrm{~g} / \mathrm{cm}^{3}$ qui n'est pas significativement différent de $\rho_{\mathrm{s}}$ déterminé en pycnométrie à gaz.

\section{Conclusion}

L'utilisation d'une pression pneumatique pour caractériser les propriétés de retrait de l'échantillon remanié, tout en faisant varier le potentiel matriciel de l'eau, aurait pour conséquence la présence d'air piégé dans les échantillons lorsque la pression n'est plus appliquée. Cette hypothèse, qui est cohérente avec les résultats expérimentaux obtenus, nous conduit à considérer la valeur de $\rho_{\mathrm{s}}$ qui en est déduite comme étant une approximation par défaut de sa valeur réelle.

\section{Remerciements}

Les auteurs remercient $H$. Gaillard (INRA, Orléans, SESCPF) pour la réalisation des expériences au cours de cette étude.

\section{Références}

Baize D. (1972) Les sols développés dans la couverture des plateaux jurassiques de Bourgogne. Pédogenèse et origine. Sci. Sol 1, 37-43

Bruand A. (1986) Contribution à l'étude de la dynamique de l'espace poral. Utilisation des courbes de retrait et des courbes de rétention d'eau. Sci. Sol 24, 4, 351-362

Bruand A. \& Prost R. (1987) Effect of water content on the fabric of a soil material : an experimental approach. J. Soil Sci., 38, 461-472

Cabidoche Y.M., Jaillard B. \& Ney B. (1985) Dynamique de l'eau dans les vertisols sur calcaires récifaux. Sol et Eau. Actes du séminaire, La Havane, Cuba, 8 - 20 avril, Orstom, Bondy, pp. 449-478

Chrétien J. (1986) Rôle du squelette dans l'organisation des sols. Conséquences sur les caractéristiques de l'espace poral de sols sur arènes et sur terrasses fluviatiles. Thèse, Dijon

Fiès J.C. (1984) Analyse de la répartition du volume des pores dans les assemblages argile-squelettes : comparaison entre un modèle d'espace poral textural et les données fournies par la porosimétrie au mercure. Agronomie 4, 9, 891-899

Haines W.B. (1923) The volume-changes associated with variations of water content in soil. J. Agric. Sci. 13, 296-310

Hillel D. (1988) L'eau et le Sol. Principes et Processus Physiques. Coll. "Pedasup" 5. Academia, Louvain-LaNeuve

Monnier G., Stengel P. \& Fiès J.C. (1973) Une méthode de mesure de la densité apparente de petits agglomérats terreux. Application à l'analyse des systèmes de porosité du sol. Ann. Agron. (Paris) 24, 5, 533-545

Reeve M.J. \& Hall D.G. (1978) Shrinkage in clayey sub- 
soils of contrasting structure. J. Soil Sci. 29, 315-323

Richards L.A. (1941) A pressure membrane extraction apparatus for soil solution. Soil Sci. 51, 377-386

Robain H. \& Curmi P. (1986) Rôle de la structure pédologique sur le comportement hydrique du sol aux faibles pF. C.R. Acad. Sci. Paris sér. II, 303, 6, 509-514

Sorani V., Bruand A., Fournier B. \& Tessier D. (1987)

Comportement des horizons d'un sol argileux en fonc- tion de l'état hydrique : étude d'échantillons à structure conservée. Sci. Sol 25, 3, 133-144

Tessier D. (1984) Etude expérimentale de l'organisation des matériaux argileux. Hydratation, gonflement et structuration au cours de la dessication et de la réhumectation. Thèse Paris 7

Violle J. (1884) Cours de Physique. Tome I. Physique Moléculaire. Masson, Paris 